\title{
Precificação e a relação com a margem de contribuição nas decisões gerenciais: um estudo de caso
}

Este artigo teve como objetivo geral evidenciar a formação do preço de venda dos itens produzidos pela panificadora. $\mathrm{O}$ estudo buscou elucidar a seguinte problemática: como a empresa define sua estratégia de formação de preço de venda? No caminho metodológico, a pesquisa enquadrou-se em relação à abordagem como qualitativa e quantitativa; em relação aos procedimentos com o estudo de caso e como técnicas de coletas de dados e análise, utilizou-se de: observação, entrevista e planilha eletrônica. Observou-se que, dentre os produtos de fabricação própria o produto com maior margem de contribuição, é o produto 2 (Biscoito 'Chipinha'), e que o produto de menor margem de contribuição é o produto 9 (Chipa). O estudo também mostrou que o produto mais vendido é o produto 11 (pão francês). Conclui-se com o estudo que, como a empresa está ativa há 16 anos, considera-se que já conquistou seu espaço e tem um número de clientes consideráveis para que ela possa se manter, e isso nos permite apontar que, a margem de contribuição dos produtos da empresa é boa. No entanto, os preços praticados ficaram abaixo dos sugeridos pelo markup.

Palavras-chave: Precificação; Margem de Contribuição; Decisões Gerenciais.

\section{Pricing and the relationship with the contribution margin in management decisions: a case study}

\begin{abstract}
This article aimed to highlight the formation of the selling price of items produced by the bakery. The study sought to elucidate the following problem: how does the company define its sales price formation strategy? In the methodological way, the research was framed in relation to the approach as qualitative and quantitative; Regarding the procedures with the case study and as techniques of data collection and analysis, we used: observation, interview and spreadsheet. It was observed that among the products of own manufacture the product with the highest contribution margin is product 2 (Biscuit 'Chipinha '), and that the product with the lowest contribution margin is product 9 (Chipa). The study also showed that the best selling product is product 11 (french bread). It is concluded with the study that, since the company has been active for 16 years, it is considered that it has already conquered its space and has a considerable number of clients to maintain it, and this allows us to point out that, the contribution margin of the company's products is good. However, the prices charged were below those suggested by the markup.
\end{abstract}

Keywords: Pricing Contribution Margin; Management Decisions.

\section{Topic: Contabilidade de Custos}

Reviewed anonymously in the process of blind peer.
Received: 10/01/2019

Approved: 11/03/2019
Tania Rodrigues dos Santos (iD

Universidade Federal de Mato Grosso do Sul, Brasil http://lattes.cnpq.br/3607680175112999

http://orcid.org/0000-0001-7285-2614

taninhad@hotmail.com

Ivan Guimarães Meirelles

Universidade Federal de Mato Grosso do Sul, Brasil http://lattes.cnpq.br/4855799065875455 igmeirelles@gmail.com

Crislaine da Silva Souza Albuquerque (iD Universidade Federal de Mato Grosso do Sul, Brasil http://lattes.cnpq.br/0571986908153914 http://orcid.org/0000-0002-4331-8815 crislaine_souza86@hotmail.com

\author{
Rafael Viana Barbosa de Paula (iD \\ Universidade Federal de Mato Grosso do Sul, Brasil \\ http://lattes.cnpq.br/5399858016225971 \\ http://orcid.org/0000-0002-5800-0738 \\ rafavianabp@gmail.com \\ Silvio Paula Ribeiro (iD \\ Universidade Federal de Mato Grosso do Sul, Brasil \\ http://lattes.cnpq.br/8666480609633926 \\ http://orcid.org/0000-0001-9169-1190 \\ spribeiro@hotmail.com \\ André Luiz Francisco \\ Universidade Federal de Mato Grosso do Sul, Brasil \\ http://lattes.cnpq.br/9816960584983983 \\ http://orcid.org/0000-0002-6727-6701 \\ admandreluiz@hotmail.com
}

Referencing this:

SANTOS, T. R.; MEIRELLES, I. G.; ALBUQUERQUE, C. S. S.; PAULA, R. V. B.; RIBEIRO, S. P.; FRANCISCO, A. L.. Precificação e a relação com a margem de contribuição nas decisões gerenciais: um estudo de caso. Revista Brasileira de Administração Científica, v.10, n.1, p.89-100, 2019. DOI: http://doi.org/10.6008/CBPC2179-684X.2019.001.0008 


\section{INTRODUÇÃO}

A cada década que se passa, as micro, pequenas e médias empresas precisam se estruturar mais financeira, contábil e mercadologicamente para sobreviver em nichos de mercado que se tornam muito mais competitivos em virtude, principalmente de fatores, vinculados aos custos e preços. É muito significativo a mudança de comportamento dos consumidores nas últimas décadas, em virtude da competitividade proporcionada pela globalização de empresas geradoras de produtos, serviços e a evolução do consumidor que sai do assento de mero comprador, para um comprador mais técnico e voltado para a relação de custobenefício.

Atrair estes consumidores e mantê-los fiéis é um grande desafio, e isso tem sido observado nos mais diversos segmentos empresariais e, entre produtos e serviços. No entanto, a ação de atratividade de consumidores não é uma fórmula tão fácil de resolver, principalmente quando se fala a respeito de custo e preço. O microempreendedor, pequeno empresário ou empresário de médio porte deve se dedicar ao conhecimento do custo de seu produto e/ou serviço para fortalecer as decisões e as avaliações necessárias para formação do preço de venda, que muito das vezes não atende somente a critérios técnicos quantitativos, mas também critérios técnicos qualitativos. De acordo com Bruni et al. (2011), "os custos representam os gastos relativos a bens ou serviços utilizados na produção de outros bens ou serviços. Portanto, estão associados aos produtos ou serviços produzidos pela entidade".

A formação de preços é uma tarefa melindrosa. Essa complexidade deve-se em virtude de que formar preços envolve diversas áreas, entre elas, marketing, economia, contabilidade de custos, finanças e clientes, que são úteis para subsidiarem as decisões dos gestores. Essas ferramentas para formação de preço podem orientar a organização para atender o cliente ao mesmo tempo em que gera o lucro desejado (MORAIS et al., 2013).

Entretanto, muitas empresas determinam seus preços com base na intuição, em contextos nada adequados de custeio e precificação e, muitos, na experiência de mercado do seu negócio, ou até mesmo de métodos pouco convencionais não reconhecidos pela Contabilidade ou Administração ou seja, não levando em conta estudos mais aprofundados e inerentes a este processo (DOLAN et al., 1998).

Além disso, há um conflito permanente entre os gestores incumbidos de aferir os custos, geralmente profissionais das áreas de finanças e contabilidade (ou controladoria), e os gestores incumbidos de satisfazer os clientes, profissionais das áreas de marketing e/ou de vendas. Este conflito entre estas áreas das organizações, com seus respectivos pontos de vista, desgasta o diálogo, não potencializa os recursos e os possíveis ganhos das empresas e leva às decisões relativas aos preços a se constituírem em acordos imperfeitos (NAGLE et al., 2003).

Existem diversas técnicas para obter a formação de preços. Dentre essas técnicas ou estratégias, podem-se destacar: a precificação baseada em custos (Markup), a precificação baseada na concorrência; e a precificação com base no valor percebido pelos clientes (MORAIS et al., 2013). A carência de acompanhamento por contadores e administradores dentro de suas respectivas competências faz com que 
a maioria das informações gerenciadas pelas micro e pequenas empresas se tornem um mero ajuntado de dados. E desta forma, a maior parte dos empresários proprietários destas organizações podem não conseguir compreender as melhoras práticas de custeio e precificação sobre seus produtos ou serviços. Nesse sentido, estudos com o propósito de abordar a margem de contribuição como mecanismo de controle e análise dos preços podem contribuir no melhor processo de gestão da empresa.

Nesta linha, Canever (2008) define o preço de venda como uma tarefa mais que importante para os gestores, essencial, pois é por meio da formação do preço de venda que a empresa começa, efetivamente, a competir no mercado cada vez mais acirrado, e a precificação competitiva, muito das vezes não deve ser associada apenas com a concorrência, mas relevantemente interrelacionada com o custeio. De acordo com o exposto esta pesquisa conduz ao seguinte problema: como a microempresa, Panificadora 'Discreta', localizada na cidade de Três Lagoas (MS), define sua estratégia de formação do preço de venda? Desta forma, o objetivo desta pesquisa foi compreender a formação do preço de venda dos itens produzidos pela panificadora bem como, o de compreender basicamente, o preço de custo.

A adoção da correta estratégia para formar os preços de venda facilita as tomadas de decisões dos gestores, dá segurança para a competitividade, possibilita adotar estratégias seguras frente ao custo e lucro (CANEVER, 2008). Tomando essa assertiva como verdade, a presente pesquisa pretendeu, em seus objetivos específicos, averiguar os métodos e estratégias de formação do preço de venda utilizado pela microempresa. A justificativa para o desenvolvimento desta pesquisa teve como objetivo primordial estruturar o trabalho afim de contribuir cientificamente com a temática. Além de proporcionar parâmetro para outras microempresas do mesmo segmento, levando em consideração a competitividade, a perpetuidade e a solvência da empresa.

A empresa em estudo, no momento, não possui um sistema digital para apuração de custos de produção, e seus gestores possuem formação técnica em contabilidade, optante pelo simples nacional, no ramo de panificação que se situa no Bairro Santa Luzia, na cidade de Três Lagoas (MS). Sua atividade é alimentícia, e seus produtos fabricados são: pães, doces, bolos, salgados; comercializam-se também na panificadora cerveja, refrigerantes, sucos, balas, chicletes, doces, sorvetes e picolés. Foi criada no ano de 2002, e é administrada pelo casal que é proprietário. Conta com um quadro de quatro funcionários que atuam em horário comercial; após este horário, são os proprietários que a administram. Por questão de solicitação, não informaremos a razão social e o seu nome de fantasia. O termo Panificadora 'Discreta' foi adotado neste trabalho para retratar o objeto de estudo.

\section{REVISÃO TEÓRICA}

\section{Panificação, custos e preço}

Para o SEBRAE (2017), o setor de panificação possui características artesanais e industriais. A panificação artesanal é aquela em que a venda ocorre diretamente ao consumidor, e que abrange as 
padarias; já a panificação industrial é voltada para o comércio atacado, e seus produtos podem ser comercializados pelas padarias e, outros estabelecimentos, como supermercados e lanchonetes.

Em termos numéricos, de acordo com o Instituto Tecnológico de Panificação e Confeitaria (ITPC), em parceria com a Associação Brasileira da Indústria de Panificação e Confeitaria (ABIP), o setor de Panificação e Confeitaria brasileiro registrou um crescimento do segmento da ordem de 3,2\% em 2017, não descontando a inflação, o que equivaleria a um faturamento de $R \$ 90,3$ bi (ABIP, 2018). O pão francês é o principal atrativo para o consumidor frequentar uma padaria. O produto está no gosto do consumidor, e é uma tradição nas refeições do brasileiro, fazendo-o voltar à loja várias vezes durante a semana (OLIVEIRA et al., 2017).

O mercado de panificação brasileiro vem mudando bastante, nos últimos anos, a variedade de produtos e serviços que as padarias e confeitarias oferecem é muito mais ampla que em décadas anteriores. De acordo com o SEBRAE (2018) o pão do dia (tipo francês) participa com $23 \%$ de todo o lucro bruto das padarias, tem uma produtividade de três a quatro vezes maiores do que a produção de confeitados. Segundo o SEBRAE (2017), a definição do preço adequado de venda de um produto ou serviço, depende do equilíbrio entre, o preço de mercado e o valor calculado em função dos custos e despesas do negócio. Para Martins (2010), o problema de decidir o preço a ser fixado não é tarefa para solução só com dados de custo. A formação do preço de venda não visa somente o aumento do faturamento da empresa, mas compreender custos associados à preço de venda para a empresa prosperar. Naquela linha,

Em relação ao processo de formação de preços, alguns dos principais objetivos podem ser apresentados, como: proporcionar a longo prazo, o maior lucro possível; permitir maximização lucrativa da participação de mercado; maximizar a capacidade produtiva, evitando ociosidade e desperdícios operacionais; maximizar o capital empregado para perpetuar os negócios de modo autossustentado.

A formação do preço de venda não visa o simples aumento do faturamento da empresa, mas a combinação de preço e volume mais lucrativo, pois faturamento maior nem sempre significa lucro maior. O preço de venda deve ser justo para o consumidor e adequado para garantir a sobrevivência da empresa. O preço de venda é, sem dúvida, a ferramenta que produz os efeitos mais intensos e imediatos. A resposta do cliente a redução de preços não deixa dúvidas quanto a isso.

Hogan et al. (2008) relata que os custos nunca devem ser o fator exclusivo para determinar o preço, embora, exercem um papel fundamental na formulação de uma estratégia de precificação. As decisões de precificação são atreladas a decisões sobre o nível de vendas, considerando que as vendas envolvem custos de produção, marketing e administração. Teoricamente, a identificação é relativamente fácil: os gastos relacionados ao processo de produção são custos, e os direcionados à administração, às vendas e aos financiamentos são despesas (MARTINS, 2010). Quanto o registro contábil dos custos,

Pode ir de uma simplificação, com a contabilidade Financeira separando custos de despesas e registrando diretamente sua passagem dos custos aos produtos acabados, ou então acompanhando no mesmo ritmo todas as etapas seguidas nos mapas e arquivos de apropriação. Na prática, quanto mais simples for o sistema de contabilização melhor, desde que a empresa mantenha adequado sistema de arquivamento dos mapas, eletrônicos ou não.

Ainda segundo Martins (2010), custos são os gastos relativos aos bens ou serviços utilizados na produção de outros bens ou serviços. Na perspectiva de Buvinich (2014), os custos obedecem a critérios específicos em suas particularidades e, desta forma, podem ser classificados em: Diretos: São os custos de 
fácil alocação aos produtos, sendo diretamente incluídos no cálculo deles. Ou seja, consiste em todos os recursos que são apropriados diretamente na produção do produto. Apresentam características de serem mensuráveis de forma objetiva; Indiretos: São os custos de difícil alocação aos produtos/serviços e necessitam de aproximação, isto é, algum critério de rateio, para serem atribuídos aos produtos.

Fixos: São custos que, em determinado período e, em certa capacidade instalada, não variam, qualquer que seja, o volume de atividade da empresa. Existem mesmo que não haja produção; e Variáveis: Seu valor total altera-se diretamente em função das atividades da empresa. Logo varia de acordo com o volume da produção. Com base nestas terminologias iniciais, a contabilidade de custos através de sistemas digitais ou de sistemas mais tradicionais se destina a fornecer informações sobre o custeio e a precificação de produtos e serviços para os diversos segmentos e níveis da administração organizacional, auxiliando estes últimos a compreenderem melhor os indicadores para tomadas de decisão, frente aos cenários que a empresa está envolvida (LEONE, 2000).

Segundo Sehnem (2011), é cada vez mais claro, no Brasil moderno e competitivo, que o mercado é o grande definidor por preço; diante disso, surge a ideia de se utilizar a margem de contribuição para auxiliar nas tomadas de decisões relativas à fixação dos preços de venda. A Margem de Contribuição é a relação entre custo, volume e lucro (CVL). O conceito Margem de Contribuição é especialmente é útil no planejamento empresarial, porque fornece informações sobre os fatores envolvidos principalmente, os correlacionados ao potencial de lucro da empresa (WARREM et al., 2003).

O cálculo da Margem de Contribuição Unitária, conforme Martins (2010), é a diferença entre o preço de venda, menos os gastos variáveis (custo variável mais gastos variáveis), sendo que, o resultado é destinado a pagar os gastos fixos (custos fixos mais gastos fixos). Observe a representação matemática: MC=PV-CV-FV, na qual MC: margem de contribuição; PV: preço de venda; CV: custo variável; e DV: despesa variável. A margem de contribuição demonstra como cada produto colabora para primeiro, amortizar os gastos fixos, e, depois, constituir o lucro esperado pelos sócios (HORNGREN et al., 2004; FERREIRA, 2007; MARTINS, 2010).

De forma genérica, a margem de contribuição é uma ferramenta muito útil porque não apropria gastos fixos ao produto, pois, utiliza o sistema de custeio variável. Assim, são apropriados apenas os gastos variáveis, ou seja, os custos alocados a cada produto são os que realmente foram gastos por ele. Assim, o gestor não terá o risco de errar por possuir informações distorcidas por rateios arbitrários, já que, a margem de contribuição elimina essa distorção (MARTINS, 2010). Conforme Zart et al. (2018), em análise de custos de linha de produtos de confeitaria, com o propósito de identificar, o markup dos produtos da linha de fabricação própria de uma panificadora, os resultados apontaram que entre os 32 produtos analisados, o Produto definido como 1 (um), apresentou a maior margem de venda (R\$22,87), enquanto o produto 5 (cinco) teve maior markup $(132,72 \%)$.

Em estudo sobre a gestão de custos para tomada de decisão, Alves et al. (2015) identificou os custos de determinados produtos e sua lucratividade, a partir da análise do setor de produção de uma panificadora. Os autores evidenciaram que o pão francês tem grande participação na receita e na lucratividade e, que o pão doce gera uma lucratividade menor. Entretanto, sua produção é viável para a panificadora. Já com 
relação aos bolos, foi comprovado que são pouco rentáveis e a produção no modo operatório atual gera prejuízos.

Com o propósito de analisar os procedimentos de custeio praticados pela empresa panificadora do município de Belo Horizonte e se a Técnica de gestão de custo utilizada mantém a relação do custo-volumelucro. Constatou-se que o método de custeio variável apresentou melhor desempenho de gestão frente ao método de Mark-up utilizado pela empresa, uma vez que a apreciação de custo dos itens que compõem a matéria prima demonstra que o valor do custo variável foi 5,79\% menor e sua MC Unitária apresentou R\$0,29 maior. Com isso, o ponto de equilíbrio em quantidade pode ser adquirido com a venda em menor quantidade do pão Frances em quilo (FERNANDES et al., 2014). No próximo tópico foram apresentados os detalhes metodológicos da pesquisa.

\section{METODOLOGIA}

As características metodológicas do trabalho foram estruturadas conforme: o objeto, o objetivo, a abordagem da pesquisa, instrumento de pesquisa e a organização dos dados para análise. Quanto à caracterização do objeto de estudo se trata de uma panificadora, aqui neste trabalho, denominada Panificadora 'Discreta', está localizada na cidade de Três Lagoas (MS), precisamente no bairro Santa Luzia da referida cidade.

Sobre a estrutura do objetivo da pesquisa, optou-se pela pesquisa exploratória, pois esta técnica permite um maior conhecimento sobre determinado fato ou evento ainda pouco estudado no objeto de estudo, além de permitir compreender as possíveis relações do objeto de estudo frente ao ambiente econômico e social que o permeia (GIL, 2010). O tipo de pesquisa realizado caracteriza-se como estudo de caso, por descrever e analisar a realidade da margem de contribuição de apenas uma empresa, não permitindo generalizar as considerações (ZART et al., 2018).

O estudo de caso é uma investigação empírica que aborda fenômeno contemporâneo, e normalmente, é adequado quando as circunstâncias são complexas e podem mudar, quando as condições que dizem respeito não foram encontradas (YIN, 2010). No caso, em questão, identificar a margem de contribuição, ou o preço de venda dos produtos, não é tarefa complexa. Porém, obter os dados reais de uma pequena empresa corresponde a uma atividade deverás complicada, muito por conta da falta de interesse dos proprietários da pequena empresa.

Para subsidiar a pesquisa, fundamentou-se na abordagem quantitativa, sob a técnica de tabulação de valores e informações referentes a Gastos Fixos e Gastos Variáveis, a instrumentalização de tais dados em tabelas garantiu uma melhor compreensão e análise na triagem, diagnóstico e parecer técnico frente ao propósito vinculado à Margem de Contribuição. Dentro dos objetivos específicos caracterizados na pesquisa está, o que procurou retratar o conhecimento sobre a margem de contribuição de produtos e margem de contribuição global da empresa frente aos seus gastos totais, auferindo no final se a empresa incorre em lucro ou prejuízo, ou se ainda precisa adequar sua política de preços e custos diante do mercado consumidor. 
O instrumento para coleta de dados utilizado no trabalho foi elaborado no software Excel, produzindo questionários e formulários, necessários à compreensão quantitativa sobre o tema abordado contextualizado por pesquisa bibliográfica, campo e documental, dirigidas estas questões, após terem sido estruturadas, aos envolvidos diretamente com a empresa como os funcionários e proprietários. Quanto à fase final de tratamento e interpretação dos dados levantados no trabalho optou-se pelo tratamento de análise matemática, instrumentalizando-se através da matemática aplicada na Contabilidade e Administração, princípios de custeio, precificação e margem de contribuição.

\section{RESULTADOS}

Nesta seção, foram apresentados os resultados encontrados na pesquisa de campo, assim como as etapas adotadas até se identificar a Margem de Contribuição, com base no rol de produtos comercializados através da produção própria da empresa. Na primeira etapa, foi necessário identificar os custos fixos e as despesas fixas, produzindo assim o somatório dos gastos totais da empresa incorridos no mês. Ainda na primeira etapa, foi necessário identificar o regime de tributação, bem como seus gastos fixos total e a margem de lucro desejada pela empresa, o que reflete em cada produto vendido. A estruturação das fórmulas trabalhadas, identificando tanto o Markup divisor, quanto multiplicador são ilustradas na tabela 1.

Tabela 1: Levantamento de Gastos Fixos Total e Formação do Markup.

\begin{tabular}{|c|c|}
\hline \multicolumn{2}{|c|}{ LEVANTAMENTO DE GASTOS FIXOS TOTAIS } \\
\hline DISCRIMINAÇÃO & VALORES \\
\hline Custos fixos & $\mathrm{R} \$ 8.000,00$ \\
\hline Despesas fixas & $\mathrm{R} \$ 7.000,00$ \\
\hline TOTAL GLOBAL & $\mathrm{R} \mathbf{1 5 . 0 0 0 , 0 0}$ \\
\hline MARKUP DIVISOR E MULTIPICADOR PARA PRECIFICAÇÃO \\
\hline Custo unitário & $100 \%$ \\
\hline Impostos & $3,2198 \%$ \\
\hline Outros gastos & $0 \%$ \\
\hline Margem de lucro & $50 \%$ \\
\hline Markup divisor & $\mathrm{R} \$ 0,4678$ \\
\hline Markup multiplicador & $\mathrm{R} \$ 2,1377$ \\
\hline
\end{tabular}

Para melhor compreensão do significado de Markup divisor ou multiplicador importa dizer que são fatores, produtos matemáticos que auxiliam o empreendedor a ter um parâmetro, para a precificação de seus itens de venda, que lhe possa assim, garantir retorno sobre os produtos comercializados com base na produção ou revenda. Na segunda etapa, em campo, foi necessário, junto aos proprietários, de se fazer o levantamento de gastos variáveis, com base na carteira de produtos produzidos pela empresa. Não foi adicionado a esta análise outros produtos do negócio como: balas, bombons, refrigerantes, sucos, etc..

Algumas considerações sobre os modelos matemáticos são observadas a seguir, no memorial de cálculo, e, posteriormente, vinculados à tabela 2. Cabe destacar que, o exemplo é com base na tabela de dados encontrados calculando somente, a primeira linha e adotando-se o mesmo, procedimento para as linhas subsequentes. 
Tabela 2: Memorial de Cálculo.

\begin{tabular}{|c|c|c|}
\hline Item & Fórmula & Exemplo \\
\hline Custo Médio Variável & Custo Variável/Qtd. Produzida & $\mathrm{R} \$ 17,00 / 10=\mathrm{R} \$ 1,70$ \\
\hline Markup multiplicador & $1 /$ markup divisor & 2,1377 \\
\hline Preço de Venda Calculado (com markup) & Custo Médio Variável x markup multiplicador & $\mathrm{R} \$ 1,70 \times 2,1377=\mathrm{R} \$ 3,63$ \\
\hline Receita (com markup) & Preço de Venda (com markup) x Qtd. Vendida & $\mathrm{R} \$ 3,63 \times 10=\mathrm{R} \$ 36,34$ \\
\hline $\begin{array}{c}\text { Preço Praticado (sem critério quantitativo, } \\
\text { mais qualitativo) }\end{array}$ & Sem análise & $\mathrm{R} \$ 3,00$ \\
\hline Receita & Preço de venda x Qtd. Vendida & $\mathrm{R} \$ 3,00 \times 10=\mathrm{R} \$ 30,00$ \\
\hline $\begin{array}{c}\text { Margem de Contribuição Unitária (com } \\
\text { markup) }\end{array}$ & $\begin{array}{c}\text { Preço de Venda (com markup) - Custo Médio } \\
\text { Variável }\end{array}$ & $\mathrm{R} \$ 3,63-\mathrm{R} \$ 1,70=\mathrm{R} \$ 1,93$ \\
\hline $\begin{array}{c}\text { Margem de Contribuição Total (com } \\
\text { Markup) }\end{array}$ & $\begin{array}{l}\text { Margem de Contribuição Unitária (com markup) x } \\
\text { Qtd. Vendida }\end{array}$ & $\mathrm{R} \$ 1,93 \times 10=\mathrm{R} \$ 19,30$ \\
\hline $\begin{array}{c}\text { Margem de Contribuição do Preço Praticado } \\
\text { (sem markup) }\end{array}$ & Preço de Venda - Custo Médio Variável & $R \$ 3,00-R \$ 1,70=R \$ 1,30$ \\
\hline $\begin{array}{l}\text { Margem de Contribuição Total (sem } \\
\text { markup) }\end{array}$ & $\begin{array}{l}\text { Margem de Contribuição Unitária (sem markup) x } \\
\text { Qtd. Vendida }\end{array}$ & $\mathrm{R} \$ 1,30 \times 10=\mathrm{R} \$ 13,00$ \\
\hline \% Participação com Preço Markup & $\begin{array}{l}\text { (Receita "com markup" / Total Global da Receita } \\
\text { "com markup") x100 }\end{array}$ & $\begin{array}{c}(\mathrm{R} \$ 36,34 / \mathrm{R} \$ 2.022,22) x \\
100=2 \%\end{array}$ \\
\hline \% Participação com Preço Praticado & $\begin{array}{l}\text { (Receita "sem markup" / Total Global da Receita } \\
\text { "sem markup") x } 100\end{array}$ & $\begin{array}{c}(\mathrm{R} \$ 30,00 / R \$ 1.690,00) x \\
100=2 \%\end{array}$ \\
\hline
\end{tabular}

Na tabela 2, para efeito de amostra e produção de informações quantitativas foram utilizados valores de 10 (dez), com suas respectivas unidades de grandeza para fabricação, receita exata, e também 10 sob suas respectivas unidades de grandeza para venda, caracterizando o princípio de negócio ideal para a empresa, ou seja, o que se produz se vende. Que fique claro que a empresa vende durante o mês muito; mais do que a quantidade aqui referenciada. Na terceira etapa, com auxílio do Software Excel, utilizado como ferramenta de produção de gráficos, resolveu-se identificar o comportamento da precificação dos itens produzidos, para assim, obter-se uma visualização rápida dos itens.

\begin{tabular}{|c|c|c|c|c|c|c|c|c|c|c|c|c|c|c|c|c|c|c|}
\hline \multicolumn{19}{|c|}{ LEVANTAMENTO DE GASTOS VARÍVEES COM BASE NA CARTEERA DE PRODUTOS DA EMPRESA } \\
\hline DESCRIC,AO & MEDIDA & \begin{tabular}{|c|} 
QTD. \\
PRODUZIDA \\
(I)
\end{tabular} & $\begin{array}{c}\text { CUSTOS } \\
\text { YARISYEEIS } \\
\text { (2) }\end{array}$ & \begin{tabular}{|c} 
CUSTO \\
MEDIOO \\
YARIAYELL \\
(2II)
\end{tabular} & $\begin{array}{c}\text { MARK.UP } \\
\text { MULIIPLIC } \\
\text { ADOR (3) }\end{array}$ & $\begin{array}{c}\text { QTD. } \\
\text { YENDDA } \\
\text { (4) }\end{array}$ & & 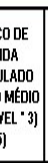 & $\begin{array}{c}\text { RECEI } \\
\text { COM PR } \\
\text { MARK-UF } \\
5\end{array}$ & 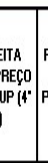 & $\begin{array}{c}\text { PRECCO DE } \\
\text { YENDA } \\
\text { PRATICAD } \\
0(6)\end{array}$ & \begin{tabular}{|c|} 
RECEIT \\
COM PRECYO \\
PRATICADO \\
$\left(4^{*} \cdot 6\right)$ \\
\end{tabular} & \begin{tabular}{|c|} 
MC UNIT. \\
PRECCO \\
COM \\
MARK-UP \\
(5- CUSTO \\
MEDIO \\
YARIAYELL) \\
\end{tabular} & \begin{tabular}{|c|} 
MC UNITI. \\
PRECOO \\
PRATICA \\
DO (6- \\
CUSTO \\
MEDIO \\
YARIAYEEL \\
\end{tabular} & \begin{tabular}{|c|} 
\\
MC TOTAL \\
PREÇO COM \\
MARK-UP \\
\end{tabular} & $\begin{array}{c}\text { MC TOTAL } \\
\text { PRECYO } \\
\text { PRATICAD } \\
0\end{array}$ & \begin{tabular}{|c|}
$\%$ \\
PARTICIP \\
ACCAO \\
COM \\
PRECKO \\
MARK-UP
\end{tabular} & \begin{tabular}{|c|}
$\%$ \\
PARTICIP \\
ACCAO \\
COM \\
PRECO \\
PRATICA \\
D0 \\
\end{tabular} \\
\hline BENNHO & UNDAREE & & 17,00 & 㬨 & 2,1377 & 10 & Ris & 3,63 & Ps & $36,34 \mathrm{~F}$ & $3,00 \mid \mathrm{F}$ & 30,00 & $\begin{array}{|ll|}\text { R } & 1,93 \\
\end{array}$ & $\begin{array}{|ll|}\text { Pis } & 1,30 \\
\end{array}$ & Fis & $4 \sqrt{\text { R }}$ & $2 \%$ & $\%$ \\
\hline BISCOTOCHPNHA & KLLOGRRMA & & \begin{tabular}{|l|l|} 
Ris & 100,000 \\
\end{tabular} & Fis & 2,1377 & 10 & Ris & 21,38 & Rs & $213,77 \mathrm{~F}$ & $18,00 \mathrm{~F}$ & 180,00 & R\$ & $\begin{array}{|ll|}\text { Bas } & 8,000 \\
\end{array}$ & Rs & \begin{tabular}{|l|l|l|}
78 \\
\end{tabular} & 11\% & 11\% \\
\hline BISCOTOTORRRDA & KLLGRAMR & & 67,00 & Ris & 2,1377 & 10 & RA & 14,32 & Fs & 143,22 & 22000 & 20,00 & 7,62 & $\begin{array}{|ll|}\text { A } & 5,3,0 \\
\end{array}$ & Ris & 2 料 & $7 \%$ & $7 \%$ \\
\hline BISCOTO VENTOLEVY & KLLOGRAMA & & 78,00 & R\$ & 2,1377 & 10 & Ris & 16,67 & Rs & $166,74 \mathrm{~F}$ & $\begin{array}{lll}R & 14,00 & F\end{array}$ & 140,00 & R\$ & $\begin{array}{|ll|}\text { Bis } & 6,20 \\
\end{array}$ & R\$ & 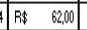 & $8 \%$ & $8 \%$ \\
\hline $\begin{array}{l}\text { BOLOCONEETRDO } \\
\text { SMPLES }\end{array}$ & KLIOGRAMA & & 156,00 & Ris & 2,1377 & 10 & Rs & 33,35 & Rs & $33,477 \mathrm{P}$ & 28,00 & 280,00 & 17,75 & Rs 12,40 & 17,47 & 78 & 19\% & $7 \%$ \\
\hline $\begin{array}{l}\text { BOLOCHOCOLATE } \\
\text { CONFETADO }\end{array}$ & KLLGRAMA & & 18,000 & R\$ & 2,1377 & 10 & Rs & 38,05 & is & 300,50 P & 32,00 & 320,00 & R\$ 20,25 & Fis 14,20 & R\$ & $\mid \begin{array}{ll}0 & 14200 \\
\end{array}$ & 19\% & $19 \%$ \\
\hline BFiGQAEERO & UNDADE & & \begin{tabular}{|l|l|} 
Ris & 17,00 \\
\end{tabular} & Fis & 2,1377 & 10 & Rit & 3,63 & Ps & $36,34 \mathrm{~F}$ & $3,00 \mathrm{~F}$ & 30,00 & R\$ & $\begin{array}{|ll|}F^{\prime} & 13,0 \\
\end{array}$ & Ris & $4 \sqrt{18}$ & $2 \%$ & $2 \%$ \\
\hline CAPOLINA & KLLGRAMA & & Ris $\quad 170,000$ & Fis & 2,1377 & 10 & Pit & 36,34 & Fis & 36,40 A & PA $30,00 \mathrm{~F}$ & 300,00 & $\begin{array}{|ll|}\text { Ris } & 19,34 \\
\end{array}$ & Af 13,000 & Fis & \begin{tabular}{lll|}
0 & 100,00 \\
\end{tabular} & $18 \%$ & $1 \%$ \\
\hline CHPA & UNDADEE & & Bis 20,00 & 变 & 2,1377 & 10 & Fis & 4,28 & Fs & $42,25 \mathrm{~F}$ & $\begin{array}{|ll|}\text { PAs } & 3,50 \\
\end{array}$ & 35,00 & R\$ & $\begin{array}{|ll|}\text { As } & 1500 \\
\end{array}$ & Ris & \begin{tabular}{|l|l|}
5 & 150,00 \\
\end{tabular} & $2 \%$ & $\%$ \\
\hline PROOEQQUENO & UNDADEE & & $\begin{array}{|ll|}\text { Ris } & 7,00 \\
\end{array}$ & Ris & 2,1377 & 10 & Pa & 1,50 & Rs & $14,96 \mathrm{~F}$ & 1.25 & 2,50 & R\$ & $\begin{array}{|ll|}\text { Bis } & 0,55 \\
\end{array}$ & R\$ & \begin{tabular}{|l|l|}
$6 \$$ \\
\end{tabular} & $1 \%$ & $1 \%$ \\
\hline PROFRANCES & KLLGGRAMR & & $\begin{array}{|ll|}\text { Pis } & 56,00 \\
\end{array}$ & 陆 & 2,1377 & 10 & PA & \begin{tabular}{ll|l}
1,97 \\
\end{tabular} & fis & $|19,71|_{\mathrm{A}}$ & $\begin{array}{lll}\text { PA } & 10,000 \\
\end{array}$ & 100,00 & R申 & $\begin{array}{|ll|}\text { Pis } & 440 \\
\end{array}$ & Ro & 行 & $6 \%$ & $\%$ \\
\hline PYOSOOYADO & UNDADE & & $\begin{array}{|ll|}\text { Ris } & 28,000 \\
\end{array}$ & Ris & 2,1377 & 10 & Pis & 5,99 & Rs & 59,956 & $5,00 \mid \mathrm{F}$ & 50,00 & R\& & $\begin{array}{|ll|}\text { fit } & 2,200 \\
\end{array}$ & RA & \begin{tabular}{|l|l|}
58 \\
\end{tabular} & $3 \%$ & $3 \%$ \\
\hline ROSCA GRANDE & UNDADEE & & Bis 45,00 & Ris & 2,1377 & 10 & PA & 9,62 & Rs & $96,19 \mathrm{~F}$ & $8,00 \mathrm{~F}$ & 80,00 & $R \$$ & $\begin{array}{|ll|}\text { Pas } & 3,500 \\
\end{array}$ & Ris & \begin{tabular}{l|l|}
$9 R \$$ \\
\end{tabular} & $5 \%$ & $5 \%$ \\
\hline ROSQUNHA & UNDADE & & Ri 7,00 & R\$ & 2,1377 & 10 & Pa & 1,50 & Fis & $14,96 \mathrm{~F}$ & $1,25 \mathrm{~F}$ & 12,50 & R\$ & \begin{tabular}{|ll} 
Pas & 0,55 \\
\end{tabular} & R\$ & 6 & $\%$ & $1 \%$ \\
\hline TOTAL GLOBAL & & 140 & Rः 946,00 & Rs & & 140 & Rs & 14,4 & R\$ 2.02 & 022,22 & \begin{tabular}{|l|l|} 
Rः & 12,07 \\
\end{tabular} & Rः $1.690,00$ & R\$ 7,69 & Rs 5,31 & R\$ $1.076,22$ & \begin{tabular}{|l|l|} 
\\
\end{tabular} & $100 \%$ & $100 \%$ \\
\hline
\end{tabular}

Figura 1: Gastos Variáveis de Produção na Carteira de Produtos Produzidos.

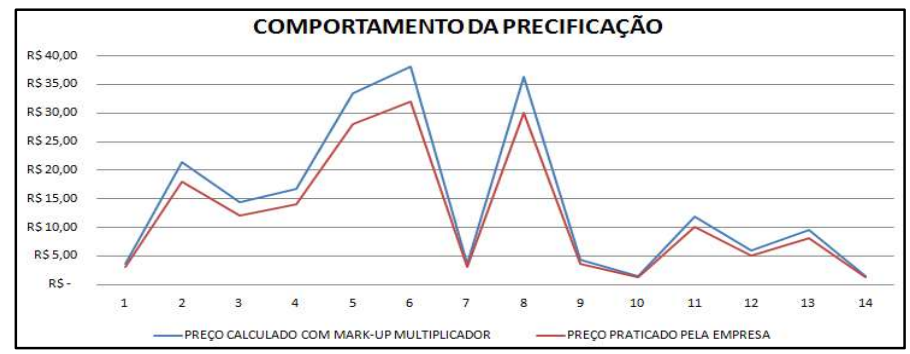

Gráfico 01: Comportamento da Precificação com e sem markup. 
No entanto, deve-se destacar que a empresa em estudo tem praticado o preço de venda abaixo do cálculo pelo markup multiplicador, o que se pode inferir, neste caso, é que o faturamento da empresa está abaixo do esperado, ou do sugerido, pela ferramenta, no comportamento da precificação. Esta situação colabora com as considerações (HORNGREN et al., 2004; FERREIRA, 2007; BRUNI et al., 2007; SPERLING, 2008; MARTINS, 2010) de que a precificação é relevante no processo de tomada de decisão. E, inclusive, sobre a posição estabelecida por (HOGAN et al., 2008; SEHNEM, 2011), onde o mercado determina o preço dos produtos, ou seja, os preços praticados pela empresa estudada são influenciados pelo mercado local. Observe a tabela 3.

Tabela 3: Receita no Ponto de Equilíbrio, com e sem markup.

\begin{tabular}{|c|c|}
\hline \multicolumn{2}{|c|}{ RECEITA NO PONTO DE EQUILíBRIO } \\
\hline DISCRIMINAÇÃO & VALORES \\
\hline Custos fixos & $\mathrm{R} \$ 0,000,00$ \\
\hline MC total (MC total com mark-up / Receita total com mark-up) & $\mathrm{R} \$ 0,4402$ \\
\hline MC total preço praticado (MC total praticado / receita total praticada) & $\mathrm{R} \$ 28.185,00$ \\
\hline Receita no ponto de equilíbrio com markup & $\mathrm{R} \$ 34.072,58$ \\
\hline
\end{tabular}

Após ter conhecimento sobre a Margem de Contribuição de cada item, identificado no rol de produtos fabricados pela empresa, partiu-se para a identificação da receita necessária da empresa no ponto de equilíbrio simulando-se a receita no ponto de equilíbrio com markup e com o preço praticado sem markup. Na quarta etapa ficou estabelecida, na figura 2, a receita necessária e a quantidade a ser vendida pela empresa durante o mês, para cobrir os gastos totais. Vale destacar que o conceito fundamental de ponto de equilíbrio significa identificar a quantidade necessária a ser vendida para que se obtenha receita, suficientemente necessária, para cobrir os gastos totais. Observe a figura 2 com sua relação matemática presente por coluna na tabela nela inserida.

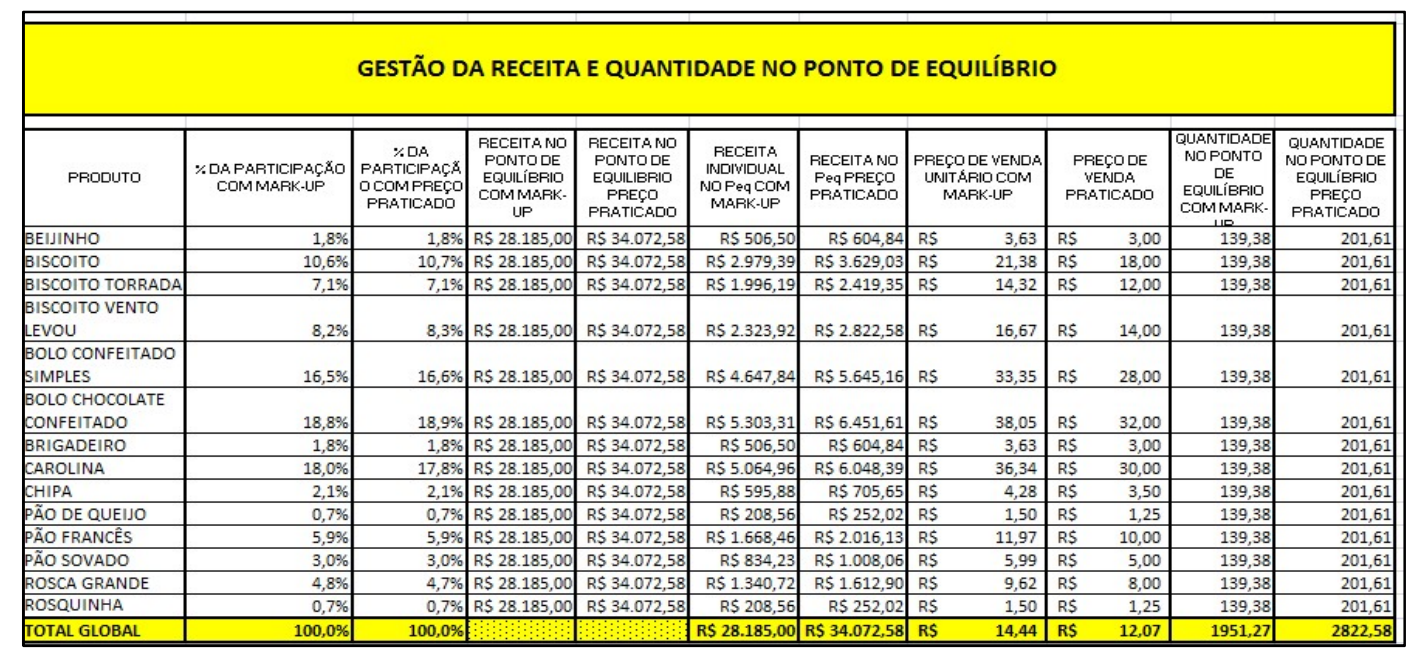

Figura 2: Receita e Quantidades Necessárias para Atingir o Ponto de Equilíbrio.

Partindo-se do princípio de apropriação dos gastos fixos total e markup (tabela 1), o método utilizado foi através da análise temporal, para se apropriar os gastos fixos total, desta forma observando a média dos registros de gastos do último semestre, o mesmo princípio foi adotado para impostos e outros gastos para a produção do markup. 
Quanto ao levantamento dos gastos variáveis com e sem markup (figura 1) fica claro uma acentuada discrepância entre o valor real com markup que deveria ser praticado e o valor de mercado utilizado pela empresa em cada item analisado vendido. Embora a empresa imagine que esteja tendo lucro de $50 \%$ sobre cada item, isso não é real, pois percebe-se que o markup utilizado computando os valores de impostos e margem de lucro 'arrastaria' o preço de cada item além do que está sendo praticado atualmente pela organização. A tabela 3 mostra o comportamento dos preços que deveriam ser praticados com o markup frente o preço praticado pela empresa com o método qualitativo. Na análise de gabinete, através de simulações, observou-se que a margem de lucro é de $41 \%$, e este valor foi comunicado para os gestores da empresa.

Por fim, observamos que caso houvesse a possibilidade de a empresa aplicar o markup encontrado, desde que não afetasse sua carteira de clientes e se mantivesse competitiva pelo preço frente a concorrência; ela teria que fazer um menor esforço de vendas em sua quantidade para alcançar o ponto de equilíbrio (figura 2). No entanto, as considerações, desta pesquisa, encontram-se com os resultados de outros estudos (ALVES, et al., 2015; FERNANDES et al., 2014; ZART et al., 2018), ao evidenciarem a relevante do cálculo da margem de contribuição e da precificação para toma de decisão.

\section{CONSIDERAÇÕES FINAIS}

O presente estudo teve como objetivo geral contribuir com a temática em questão, e também, com as pequenas empresas, na compreensão quantitativa da formação do custeio e preço de venda de seus produtos, empregando como técnica de precificação sobre o gasto variável, o fator de markup multiplicador, instrumento norteador para a precificação.

Na saída de campo, pode-se observar que a empresa, embora tenha controle sobre os Gastos Fixos Total, e também, o controle sobre o gasto variável sobre, cada receita utilizada na produção dos itens de venda na padaria, a gestão da empresa não utiliza meios quantitativos para precificar seus produtos, mas procuram compreender de forma qualitativa como os concorrentes e mercado em que atua vendem e absorva os produtos, idealizando desta forma o modelo de competitividade mais adequada para a gestão da empresa.

O que fica observado, após o levantamento de campo dos dados levantados e aplicando técnicas e conceitos matemáticos, contábeis e de administração, é que a empresa incorre em alguns erros que aparentemente podem estar minimizando, a lucratividade da empresa em longo prazo. Em relação, à maximização de recursos financeiros na computação de gastos totais e impostos devem ser acompanhados fidedignamente pelos gestores, pois refletem diretamente na necessidade de quantidade e receita a serem produzidas pela empresa a fim de se alcançar o ponto de equilíbrio.

Portanto, como recomendação à organização após este estudo aconselhou-se aos gestores que tenham atenção a geração de gastos fixos, pois estes podem significativamente corroer a lucratividade da empresa, bem como contribuir para maior esforço da empresa em vendas para cobrir estes gastos. Análises de simulação com auxílio do software Excel alimentado com as fórmulas utilizadas no contexto científico para 
cálculo de identificação do markup, margem de contribuição e ponto de equilíbrio demonstra que a empresa pratica seus preços abaixo do estabelecido que é $50 \%$.

A recomendação dada neste quesito aos gestores e proprietários da empresa é que, sabendo que embora a empresa esteja tendo lucratividade, o que foi comunicado por eles, o ideal é que se realmente quisessem ter lucratividade de 50\% sobre cada item deveriam aplicar o markup corretamente, mas desde que estes itens após fossem mudados não ficassem fora do mercado concorrente.

Ficou claro que produtos fora do preço competitivo podem reduzir o volume de vendas e desta forma deixar a empresa dependente de fontes de financiamento para pagar gastos fixos ou até mesmo variáveis. A política da empresa para momento futuro é o de aumentar a lucratividade de $50 \%$ para $80 \%$, conforme comunicado pelos gestores, após este estudo desaconselhamos os gestores a praticarem o aumento na lucratividade, mas o de reduzir gastos variáveis e gastos fixos no intuito de melhorar a estratégia de lucro. Em síntese concluímos que os modelos matemáticos, contábeis e de administração, quando bem aplicados frente ao objeto de estudo, puderam trazer informações relevantes para uma gestão mais eficiente da empresa.

\section{REFERÊNCIAS}

ABIP. Associação Brasileira da Indústria de Panificação e Confeitaria. Balanço e tendência do mercado de panificação e confeitaria. Brasília: ABIP, 2018.

ALVES, L. V.; SILVA, V. L.; CARDOSO, R.; LUCAS, R. E. C.; JUNIOR, J. F. R.. Gestão de custos para tomada de decisão: um estudo de caso em uma panificadora na cidade de João Pessoa. In: ENCONTRO NACIONAL DE ENGENHARIA DE PRODUÇÂO, 25. Anais. Fortaleza: 2015.

BRUNI, A. L.; FAMÁ, R.. Gestão de custos e Formação de Preços. 5 ed. São Paulo: Atlas, 2011.

BRUNI, A.; FAMÁ, R.. Gestão de Custos e Formação de Preços. 4 ed. São Paulo: Atlas, 2007.

BUVINICH, M. R.. Apostila sobre custos Industriais. João Pessoa: UFPB, 2014.

CANEVER, P. F.. Estratégia de formação de preço de venda em indústrias de Santa Catarina. Florianópolis, 2008.

DOLAN, R. J.; SIMON, H.. O poder dos preços: as melhores estratégias para ter lucro. São Paulo: Futura, 1998.

FERNANDES, D. S. M.; PEREIRA, F. S.; SANTOS, E. E.. Custo para tomada de decisão: um estudo de caso em uma empresa panificadora do município de Belo Horizonte. Belo Horizonte: UNIBH, 2014.

FERREIRA, J. A. S.. Contabilidade de Custos. São Paulo: Pearson Prentice Hall, 2007.

GIL, A. C.. Como elaborar projetos de pesquisa. 5 ed. São Paulo: Atlas, 2010.
HOGAN, E. J.; NAGLE, T. T.. Estratégias e táticas de preço: um guia para crescer com lucratividade. São Paulo: Pearson Prentice Hall, 2008.

HORNGREN, C. T.; SUNDEM, G. L.; STRATTON, W. O.. Contabilidade Gerencial. 12 ed. São Paulo: Pearson Prentice Hall, 2004.

LEONE, G. S. G.. Curso de contabilidade de custos. 2 ed. São Paulo: Atlas, 2000

MARTINS, E.. Contabilidade de custos. São Paulo: Atlas, 2010

MORAIS, F. J.; VIEIRA, P. E.. Estratégia de precificação para empresas comerciais. Ijuí: UNIJUÍ, 2013.

NAGLE, T. T.; HOLDEN, R. K.. Estratégia e tática de preços: um guia para decisões lucrativas. 3 ed. São Paulo: Pearson Prentice Hall, 2003.

OLIVEIRA, M. R. D.; VIANA, M. M.. 20: Norma ABNT do Pão Tipo Francês. Brasília: SEBRAE, 2017.

SEBRAE. Preço: Como definir o preço de venda de um produto ou serviço. Brasília: SEBRAE, 2017.

SEBRAE. Serviço Brasileiro de Apoio às Micro e Pequenas Empresas. Ideias de negócios: Como montar uma padaria. Brasília: SEBRAE, 2017.

SEBRAE. Serviço Brasileiro de Apoio às Micro e Pequenas Empresas. $\mathbf{O}$ diferencial financeiro do Pão do Dia. Brasília: SEBRAE, 2018

SEHNEM, M. A.. Formação de preços de venda e sua flexibilidade em empresas de transportes de cargas. Caxias do Sul: 2011. 
SPERLING, E.. A influência da formação do preço de venda na micro e pequena empresa do comércio varejista nos relatórios gerenciais. Revista Interdisciplinar Científica Aplicada, Blumenau, v.2, n.1, p.1-18, 2008.

WARREN, C. S.; REEVE, J. M.; FESS, P. E.. Contabilidade Gerencial. São Paulo: Thomson Learning, 2003.
YIN, R. K.. Estudo de caso: planejamento e métodos. 4 ed. Porto Alegre: Bookman, 2010.

ZART, F. C. A.; ZART, C. M.; ZANATTA, M. J.. Análise de custo de linha de produção de confeitaria em uma panificadora. Revista Livre de Sustentabilidade e Empreendedorismo, v.3, n.2, p.20-47, 2018.

A CBPC - Companhia Brasileira de Produção Científica (CNPJ: 11.221.422/0001-03) detém os direitos materiais desta publicação. Os direitos referem-se à publicação do trabalho em qualquer parte do mundo, incluindo os direitos às renovações, expansões e disseminações da contribuição, bem como outros direitos subsidiários. Todos os trabalhos publicados eletronicamente poderão posteriormente ser publicados em coletâneas impressas sob coordenação da Sustenere Publishing, da Companhia Brasileira de Produção Científica e seus parceiros autorizados. Os (as) autores (as) preservam os direitos autorais, mas não têm permissão para a publicação da contribuição em outro meio, impresso ou digital, em português ou em tradução. 